\title{
Mechanisms of cigarette smoke induced increased airspace permeability
}

\author{
X Y Li, I Rahman, K Donaldson, W MacNee
}

\begin{abstract}
Background - Increased epithelial permeability of the airspaces occurs commonly in the lungs of cigarette smokers. It is likely to be important in augmenting the inflammatory response in the airspaces and hence may have a role in the pathogenesis of emphysema. It has previously been shown that intratracheal instillation of cigarette smoke condensate induces increased epithelial permeability in vivo in rats and in vitro in epithelial cell monolayers, associated with a disturbance in the lung antioxidant, glutathione (GSH). The aim of this study was to assess the role of neutrophils, GSH, and tumour necrosis factor (TNF) in the increased epithelial permeability following intratracheal instillation of cigarette smoke condensate.
\end{abstract}

Methods - Epithelial permeability of the airspaces was measured in rat lungs as the passage of intratracheally instilled 125iodine labelled bovine serum albumin (BSA) into the blood. The permeability of a monolayer of human type II alveolar epithelial cells to ${ }^{125}$ I-BSA was also measured.

Results - Cigarette smoke condensate produced a $59.7 \%$ increase in epithelial permeability over control values peaking six hours after instillation and returning to control values by 24 hours. Depletion of neutrophils and, to a lesser extent, macrophages by an intraperitoneal injection of antineutrophil antibody did not influence the increased epithelial permeability induced by cigarette smoke condensate. Although instillation of human recombinant $\mathrm{TNF} \alpha$ produced an increase in epithelial permeability in the rat lung from 0.62 $(0.61) \%$ to $1.27(0.08) \%$, only a trivial amount of TNF $\propto$ was detected in bronchoalveolar lavage (BAL) fluid in vivo or in culture medium from BAL leucocytes obtained from animals treated with cigarette smoke condensate $(94.9(28 \cdot 8)$ units $/ \mathrm{ml})$. Furthermore, antiTNF antibody did not abolish the increased epithelial permeability produced by cigarette smoke condensate. The role of GSH was assessed by measuring the changes in both the reduced (GSH) and oxidised form (GSSG) in lung tissue and in BAL fluid. One hour after instillation of cigarette smoke condensate there was a marked fall in the GSH content in the lung (from $809.8(31.8)$ to $501.7(40.5) \mathrm{nmol} / \mathrm{g})$ in association with increased GSSG levels (from 89.8 $(2 \cdot 7)$ to $148 \cdot 7(48 \cdot 8) \mathrm{nmol} / \mathrm{g})$. This was followed by a return of GSH levels to control values, with a concomitant decrease in GSSG levels six hours after instillation. GSH levels in BAL fluid fell dramatically following cigarette smoke condensate (from $2.56(0.30)$ to $0.31(0.21) \mathrm{nmol} / \mathrm{ml})$ and this fall was sustained up to six hours after instillation of cigarette smoke condensate. Conclusions - These studies suggest that neutrophils and TNF do not have a major role in the increased epithelial permeability induced by cigarette smoke condensate. However, the data support a role for the depletion of the antioxidant glutathione in the increased epithelial permeability caused by cigarette smoke condensate.

(Thorax 1996;51:465-471)

Keywords: epithelium, neutrophils, tumour necrosis factor, glutathione.

The alveolar epithelium provides a barrier to the passage of various substances across the alveolar-capillary membrane. ${ }^{1}$ It is well established that exposure to cigarette smoke increases epithelial permeability in animals ${ }^{23}$ and in humans. ${ }^{45}$ This increase in epithelial permeability may be important, not only by allowing exudation of proteins into the airspaces as part of the inflammatory response, but also by enabling the products of inflammatory leucocytes such as proteases and smoke-derived toxins to gain access and cause damage to the lung interstitium. The mechanism of the increased epithelial permeability induced by cigarette smoke is unknown. Oxidants in cigarette smoke may play a major part in cigarette smoke-induced lung injury ${ }^{6}$ and have been implicated in the pathogenesis of emphysema. ${ }^{78}$ Glutathione (GSH) is a tripeptide which acts as an intracellular and extracellular antioxidant by supplying reducing equivalents in the form of electrons which reduce peroxides. ${ }^{9}$ Many studies have suggested that there is a re- 
lationship between the concentration of GSH in cells and their susceptibility to oxidant injury. ${ }^{910}$ In a previous study we have shown that cigarette smoke condensate decreased intracellular GSH levels in type II alveolar epithelial cells in vitro, and that this was associated with an increase in the permeability of an epithelial cell monolayer. ${ }^{11}$ Furthermore, extracellular glutathione was protective against this effect.

The role of neutrophil sequestration and migration in relation to changes in epithelial permeability is controversial. Both Kleeberger and Hudak ${ }^{12}$ and Bassett and coworkers ${ }^{13}$ reported that the influx of neutrophils and the change in epithelial permeability induced by exposure to ozone, a component of cigarette smoke, are not mutually dependent events. However, Bhalla and colleagues ${ }^{14}$ have published data which show that depletion of neutrophils prevents ozone-induced epithelial permeability.

Tumour necrosis factor (TNF) has a broad spectrum of biological activities which influence the outcome of inflammatory responses. ${ }^{15}$ It has been shown to induce increased permeability of epithelial cell monolayers, ${ }^{16}$ and a reduction in TNF activity, in association with impaired pulmonary defences caused by cigarette smoke, has been implied. ${ }^{17}$ The purpose of this study was to investigate the role of GSH, neutrophils, and the TNF as possible factors in the induction of increased alveolar epithelial permeability produced by cigarette smoke condensate.

\section{Methods \\ RADIOLABELLING OF BOVINE SERUM ALBUMIN (BSA) WITH 125-LABELLED IODINE $\left({ }^{125} \mathrm{I}\right)$ \\ Bovine serum albumin (BSA, Sigma, Poole, UK) was iodinated according to a previously described method. ${ }^{11}$ In brief, BSA was labelled with 125-iodine (Amersham International, Amersham, UK) by chloramine T. ${ }^{125} \mathrm{I}-\mathrm{BSA}$ was separated from free ${ }^{125} \mathrm{I}$ by chromatography on a Sephadex G25 column (Pharmacia, Mil- ton Keynes, UK). The efficiency of the radio- labelling was checked routinely by precipitation with $20 \%$ chloroacetic acid in the presence of fetal bovine serum and the binding rate of ${ }^{125} \mathrm{I}$ - BSA was between $85 \%$ and $90 \%$.}

INTRATRACHEAL INSTILLATION OF CIGARETTE SMOKE CONDENSATE AND INTRAPERITONEAL INJECTION OF ANTINEUTROPHIL ANTIBODY Wistar-derived rats of the HAN strain weighing 250-350 g were used. Rats were anaesthetised by an intraperitoneal injection of sodium pentobarbitone. Cigarette smoke condensate was produced by blowing smoke, generated by a smoking machine, from three medium tar cigarettes over $3 \mathrm{ml}$ of phosphate buffered saline (PBS) in a tonometer. The tracheas of anaesthetised rats were exposed. Through a small incision a blunt needle was introduced and $0.2 \mathrm{ml}$ of cigarette smoke condensate was instilled into the distal airspaces, followed by $0.5 \mathrm{ml}$ of air to aid distribution. Control rats were given $0.2 \mathrm{ml}$ of PBS through the same route. The incision was closed with a clip and the rats recovered with no ill effects. Animals were sacrificed one, four, six, and 24 hours after instillation.

To deplete circulating neutrophils, $0.9 \mathrm{ml}$ of rabbit anti-rat neutrophil serum (Accurate Chemical \& Scientific Corporation, Westbury, New York, USA) was injected into the rat peritoneal cavity and cigarette smoke condensate was given 16 hours thereafter. The numbers of leucocytes in the bronchoalveolar lavage (BAL) fluid and epithelial permeability were measured six hours after instillation of cigarette smoke condensate.

\section{MEASUREMENT OF RAT LUNG EPITHELIAL} PERMEABILITY

Airspace epithelial permeability to intratracheally instilled ${ }^{125} \mathrm{I}-\mathrm{BSA}$ was measured as previously described. ${ }^{116}$ In brief, the femoral vein of an anaesthetised rat was exposed and a G25 butterfly cannula was inserted. This was followed by an injection of $0.2 \mathrm{ml}$ of 100 units/ $\mathrm{ml}$ heparin. A blunt ended needle was inserted into the trachea and $0.1 \mathrm{ml}$ of ${ }^{125} \mathrm{I}$-BSA solution $(15 \mu \mathrm{Ci})$ was instilled intratracheally. Five minutes after instillation of ${ }^{125} \mathrm{I}$-BSA a $0.1 \mathrm{ml}$ blood sample was withdrawn from the femoral vein. This was repeated on five occasions at two minute intervals. Before each sample a mixture of blood and heparin solution was withdrawn and discarded. Each sample was then followed by an injection of $0.2 \mathrm{ml}$ heparin. The ${ }^{125} \mathrm{I}$ counts in each sample were measured in a gamma counter (Cobra II, Auto-Gamma Counting System, Meridan, USA). The proportion of BSA-bound ${ }^{125} \mathrm{I}$ was assessed in the blood samples by trichloroacetic acid (TCA) precipitation and centrifugation. ${ }^{125} \mathrm{I}$ counts in the blood samples were corrected for free ${ }^{125} I$ counts. Epithelial permeability in the rat lung was expressed as the percentage of the ${ }^{125} \mathrm{I}-\mathrm{BSA}$ counts originally instilled which were present in the whole blood volume of the rat $\left(\%{ }^{125} \mathrm{I}-\mathrm{BSA}\right.$ recovered). The blood volume of each rat was estimated from standard tables. ${ }^{18}$ Epithelial permeability was expressed as the mean values of the pooled blood samples. We have used a technique which assesses epithelial permeability as the passage of a radiolabelled protein from the airspace to the blood since similar techniques have been used in studies of epithelial permeability in smokers. ${ }^{14}$

\section{BRONCHOALVEOLAR LAVAGE IN THE RAT}

Rats were sacrificed by an overdose of sodium pentobarbitone and the trachea was cannulated as described above with a blunt needle. The lungs were removed from the thorax and bronchoalveolar lavage was carried out by instilling and withdrawing $8 \mathrm{ml}$ of saline at $37^{\circ} \mathrm{C}$ from the lungs via the cannula. The procedure was repeated four times. To assess TNF activity in BAL fluid, $4 \mathrm{ml}$ of MEM medium plus $0 \cdot 2 \%$ low endotoxin BSA (Sigma, Poole, UK) was injected and withdrawn before the normal lavage procedure. The medium was used to make 
BAL samples comparable to cell culture supernatant in later experiments.

Leucocytes in the BAL fluid were spun down and resuspended in MEM medium with $0.2 \%$ BSA. The cells were cultured overnight in 24well plates at a concentration of $1 \times 10^{6}$ cells/ $\mathrm{ml}$. The culture supernatant was obtained by spinning the medium at $1200 \mathrm{~g}$ for five minutes.

\section{MEASUREMENT OF TNF ACTIVITY}

TNF activity in BAL fluid and cultured leucocyte supernatant was detected by the standard L929 cell bioassay ${ }^{19}$ which we have previously shown to be a sensitive and specific assay for TNF, ${ }^{16}$ having a lower limit of detection of one unit of TNF. L929 cells suspended in MEM medium plus $10 \%$ FCS at $0.3 \times 10^{6} / \mathrm{ml}$ were seeded into microtitre plates and formed a monolayer after 16 hours incubation at $37^{\circ} \mathrm{C}$ in $5 \%$ carbon dioxide. The medium was then changed to MEM medium plus 5\% FCS without antibiotics and $1 \mu \mathrm{g} / \mathrm{ml}$ of actinomycin D (Sigma, Poole, UK) was added. The samples were serially diluted in microtitre plates using a multichannel pipette. Viable cells were stained with crystal violet methanol solution and $100 \mu \mathrm{l}$ of $20 \%$ acetic acid was added to each well to solubilise the stain. The plates were then read in a plate reader (Dynatech Laboratories Inc, USA). TNF activity was determined by comparison with recombinant $\mathrm{TNF} \alpha$ standards (Genzyme, Kent, UK). The activity of TNF which caused $50 \%$ killing of L929 cells was calculated and expressed as one unit.

The specificity of the L929 assay is shown by the fact that it is calibrated against recombinant human TNF and that the activity could be abolished by TNF antibody ${ }^{16}$ which was kindly supplied by Dr Joseph Fantone, Ann Arbor, Michigan, USA. In some experiments TNF antibody was injected intraperitoneally at the same time as cigarette smoke condensate was instilled.

\section{MEASUREMENT OF REDUCED (GSH) AND OXIDISED GLUTATHIONE (GSSG)}

To measure GSH/GSSG the pulmonary circulation of rat lungs was perfused with saline and bronchoalveolar lavage was performed as described above. The lungs were then carefully separated from the other tissues, blotted, weighed, and placed in a universal container. Five $\mathrm{ml}$ of $6.6 \%(\mathrm{w} / \mathrm{v})$ TCA was added and the lung tissue was homogenised. This was repeated and a further $5 \mathrm{ml}$ of TCA was added. The suspension was then centrifuged at $1200 \mathrm{~g}$ for five minutes. The supernatant was neutralised with $\mathrm{NaHCO}_{3}$ until the $\mathrm{pH}$ was $7 \cdot 0$.

The total cellular GSH concentration was assayed by the GSSG-reductase-DTNB recycling procedure described by Tietze and coworkers. ${ }^{20}$ In brief, to $100 \mu$ laliquots of neutralised supernatants were added $200 \mu \mathrm{l}$ of $100 \mathrm{mM}$ potassium phosphate, $5 \mathrm{mM}$ EDTA buffer ( $\mathrm{pH} \mathrm{7.5),} 100 \mu \mathrm{l}$ of DTNB ( $5 \mathrm{mM})$, and $100 \mu \mathrm{l}$ of GSSG reductase (10 units $/ \mathrm{ml})$. After incubation for one minute at $25^{\circ} \mathrm{C}, 100 \mu \mathrm{l}$ NADPH $(2.4 \mathrm{mM})$ was added and made up

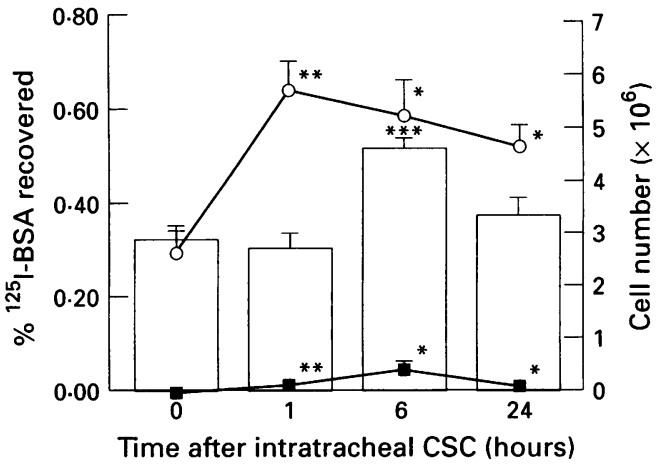

Figure 1 Epithelial permeability and components of bronchoalveolar lavage (BAL) leucocytes $(O$, macrophages; $\square$, neutrophils) in the rat lung at various times after intratracheal instillation of whole cigarette smoke condensate (CSC). Epithelial permeability is calculated as the percentage of ${ }^{125} I-B S A$ (bovine serum albumin) instilled intratracheally which appears in blood (\% ${ }^{125} I-B S A$ recovered) at the times indicated on the $x$ axis from pooled blood samples. Each point represents the mean and the bars the $S E$ of six experiments in rats. ${ }^{*} p<0.05, * * p<0.01$ compared with control values.

to a final volume of $1 \mathrm{ml}$ by the addition of phosphate-EDTA buffer. The linear increase in the absorbance at $412 \mathrm{~nm}$ was measured over two minutes at $25^{\circ} \mathrm{C}$ using a spectrophotometer (Unicam 8700 series, Cambridge, UK). The GSH or GSSG concentration was determined by comparison with a GSH standard curve. For the GSSG assay, $1 \mathrm{ml}$ of a neutralised sample was treated with 2-vinylpyridine and triethanolamine as described by Griffith ${ }^{21}$ and the treated supernatant was used in the assay.

\section{STATISTICAL ANALYSIS}

Data are presented as means (SE). Comparison between mean values was made by a one-way analysis of variance using Tukey's method.

\section{Results}

EPITHELIAL PERMEABILITY AND LEUCOCYTES IN BAL FLUID FROM RAT LUNGS INSTILLED WITH CIGARETTE SMOKE CONDENSATE Whole cigarette smoke condensate induced an increase in rat lung epithelial permeability to ${ }^{125}$ I-BSA six hours after intratracheal instillation which had resolved by 24 hours (fig 1 ). This was associated with the recruitment of macrophages and neutrophils into the BAL fluid (fig 1 ). The macrophage numbers in BAL fluid peaked one hour after instillation of cigarette smoke condensate, whereas the peak increase of neutrophils occurred at six hours. Since the lymphocyte numbers remained at very low levels and were not affected by various treatments, these data are not presented.

\section{ROLE OF NEUTROPHILS IN INCREASING} EPITHELIAL PERMEABILITY

There was an almost total depletion of circulating neutrophils 16 hours after the intraperitoneal injection of the antineutrophil serum in both control rats (from 35 (4)\% to 1 (1)\%, $\mathrm{n}=4$ ) and those exposed to cigarette smoke condensate (from 45 (3) $\%$ to $4(3) \%, n=4$ ). 


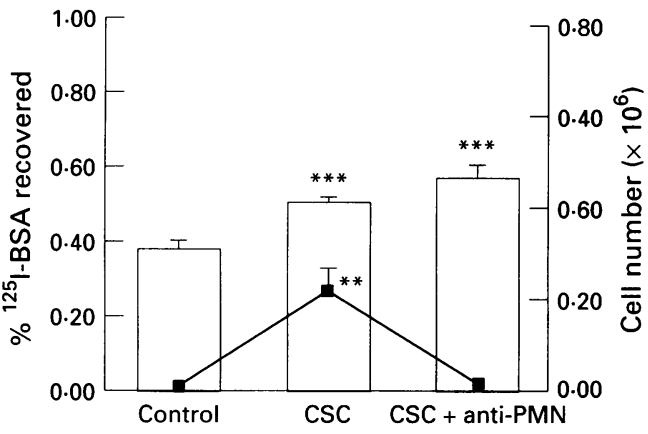

Figure 2 Effect of neutrophil antibody (anti-PMN) on cigarette smoke condensate (CSC)-induced epithelial permeability and bronchoalveolar lavage (BAL) neutrophils ( $\mathrm{D}$ in the rat lung. Epithelial permeability is expressed as the mean percentage of ${ }^{125} I-B S A$ (bovine serum albumin) counts appearing in blood from pooled blood samples (\% ${ }^{125} I-B S A$ recovered). Each point or histogram represents the mean and the bars the SE of 3-6 experiments in rats. ${ }^{* *} p<0.01, * * * p<0.001$ compared with control values.

The relationship between neutrophil depletion and epithelial permeability induced by the instillation of cigarette smoke condensate was therefore studied 16 hours after injection of the antibody. The increase in mean neutrophil numbers in BAL fluid six hours after intratracheal instillation of cigarette smoke condensate was abolished by pretreatment with antineutrophil serum and macrophage numbers in BAL fluid were also reduced (control, $3.54(0.47) \times 10^{6}$; cigarette smoke condensate alone, $3.51(0.61) \times 10^{6}$, cigarette smoke condensate plus antineutrophil serum, 2.46 $\left.(0.86) \times 10^{6} ; n=6, p<0 \cdot 05\right)$. The depletion of neutrophils in BAL fluid did not, however, alter the increased epithelial permeability caused by cigarette smoke condensate (fig 2).

ROLE OF TUMOUR NECROSIS FACTOR (TNF)

TNF activity in BAL fluid or released from leucocytes in BAL fluid obtained from animals treated by instillation of cigarette smoke condensate was not significantly increased compared with control animals (table 1) nor in controls which had intratracheal PBS (data not shown). Indeed, the levels of TNF released by BAL leucocytes were lower six and 24 hours after instillation of cigarette smoke condensate (table 1). Rat lung epithelial permeability was higher following the intratracheal instillation of $5 \times 10^{4}$ units of TNF $\alpha$ compared with control values (fig 3). A slight increase in epithelial permeability was present at eight hours, but a

Table 1 Mean (SE) tumour necrosis factor (TNF) activity in bronchoalveolar lavage (BAL) fluid and released by $B A L$ leucocytes after intratracheal instillation of cigarette smoke condensate (CSC)

\begin{tabular}{lll}
\hline $\begin{array}{l}\text { Time after } \\
\text { CSC } \\
\text { (hours) }\end{array}$ & $\begin{array}{l}\text { TNF in BAL } \\
\text { fluid } \\
\text { (units/ml) }\end{array}$ & $\begin{array}{l}\text { TNF released } \\
\text { by BAL leucocytes } \\
\text { (units/ml) }\end{array}$ \\
\hline 0 & $8 \cdot 4(4 \cdot 3)$ & $51 \cdot 6(16 \cdot 7)$ \\
1 & $20 \cdot 7(8 \cdot 6)$ & $94 \cdot 9(28 \cdot 8)$ \\
6 & $9 \cdot 2(5 \cdot 5)$ & $20 \cdot 8(5 \cdot 90)^{*}$ \\
24 & $6 \cdot 5(3 \cdot 7)$ & $13 \cdot 6(3 \cdot 7)^{*}$ \\
\hline Results are mean (SE) of 12 experiments. \\
${ }^{*}$ p<0.05 compared with time zero.
\end{tabular}

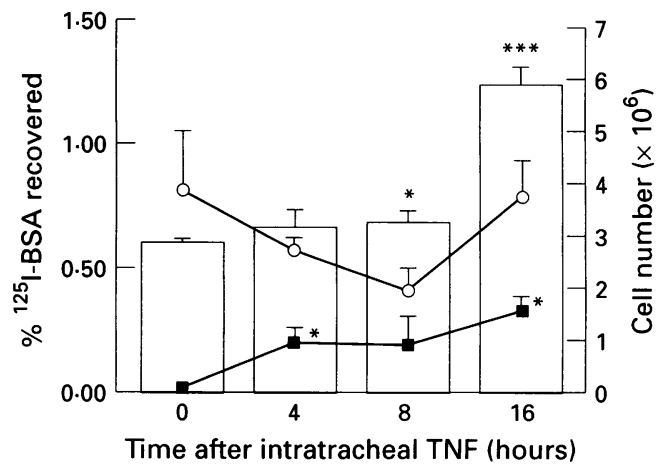

Figure 3 Epithelial permeability and the components of $B A L$ leucocytes (O, macrophages; $\square$, neutrophils) in the rat lung induced by intratracheal instillation of human recombinant TNF $\alpha\left(5 \times 10^{4}\right.$ units $)$. Each histogram and point represent mean values and the bars the $S E$ of three experiments in rats. ${ }^{*} p<0.05, * * * p<0.001$ compared with control values.

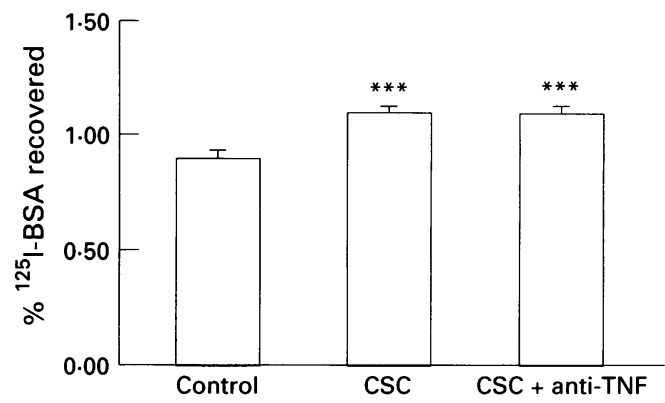

Figure 4 Effect of pretreatment with TNF antibody (anti-TNF) on the increased epithelial permeability which occurs six hours after instillation of cigarette smoke condensate (CSC) into the rat lung. Each histogram represents the mean and the bars the SE for three animals. $* * * p<0.001$ compared with control.

more significant increase occurred 16 hours after TNF instillation. An increased number of neutrophils appeared in the BAL fluid after intratracheal instillation of TNF (fig 4). However, co-instillation of cigarette smoke condensate with TNF antibody did not diminish the increase in epithelial permeability induced by cigarette smoke condensate (fig 4 ). In addition, administration of antineutrophil antibody in animals treated with cigarette smoke condensate did not significantly change TNF activity in BAL fluid nor in BAL leucocyte supernatant (table 2).

Table 2 Mean (SE) tumour necrosis factor (TNF) activity in bronchoalveolar lavage (BAL) fluid and released by $B A L$ leucocytes in culture

\begin{tabular}{lll} 
& $\begin{array}{l}\text { TNF in } \\
\text { BAL fluid } \\
\text { (units/ml) }\end{array}$ & $\begin{array}{l}\text { TNF released } \\
\text { by BAL leucocytes } \\
\text { (units/ml) }\end{array}$ \\
\hline Control & $0 \cdot 4(0 \cdot 2)$ & $36 \cdot 2(6 \cdot 1)$ \\
CSC & $0 \cdot 3(0 \cdot 2)$ & $25 \cdot 1(11 \cdot 8)^{*}$ \\
CSC + anti-PMN & $0 \cdot 1(0 \cdot 0)^{*}$ & $35 \cdot 6(19 \cdot 1)$
\end{tabular}

Results are mean (SE) from 6-8 animals.

$\mathrm{CSC}=6$ hours after intratracheal instillation of cigarette smoke condensate; CSC + anti-PMN =CSC instilled 16 hours after intraperitoneal injection of antineutrophil antibody. ${ }^{*} \mathrm{p}<0.05$ compared with control values. 


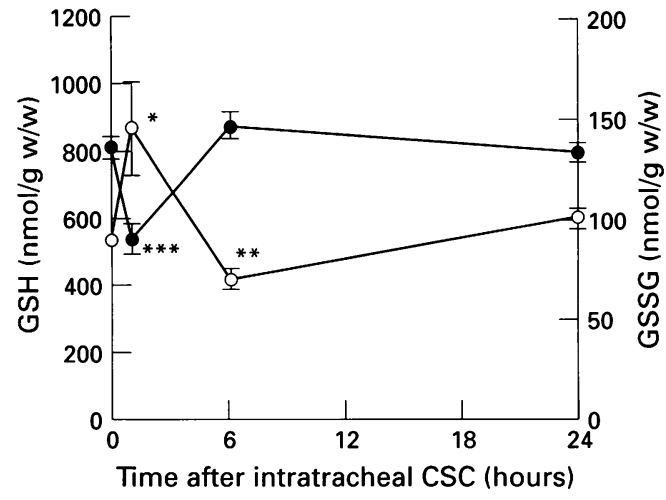

Figure 5 Reduced glutathione (GSH; O) and oxidised glutathione (GSSG; O) in the rat lung at various times after intratracheal instillation of cigarette smoke condensate (CSC). Each point represents the mean and the bars the $S E$ of 3-6 rats. $*_{p}<0.05 * * p<0 \cdot 01, * * * p<0 \cdot 001$ compared with time zero.

Table 3 Mean (SE) concentration of glutathione (GSH) in bronchoalveolar lavage (BAL) fluid after intratracheal instillation of cigarette smoke condensate (CSC)

\begin{tabular}{ll}
\hline Time after CSC (hours) & GSH $(\mathrm{nmol} / \mathrm{ml})$ \\
\hline 0 & $2.56(0 \cdot 91)$ \\
1 & $0.31(0 \cdot 21)^{* * *}$ \\
6 & $1.22(0 \cdot 20)^{* *}$ \\
24 & $3.40(1 \cdot 54)$ \\
\hline
\end{tabular}

Results are mean (SE) of 6-9 experiments.

${ }_{* *} p<0.01,{ }^{* * *} \mathrm{p}<0.001$ compared with zero hours.

RELATIONSHIP BETWEEN GSH CONTENT AND EPITHELIAL PERMEABILITY

Lung GSH content was significantly lower one hour after instillation of cigarette smoke condensate and was associated with an increase in GSSG compared with control lungs. This decrease in GSH returned to control values by six hours with the GSSG content returning to control values by 24 hours (fig 5).

Furthermore, compared with control values the concentration of GSH in the BAL fluid was dramatically lower one hour and six hours after intratracheal instillation of cigarette smoke condensate (table 3 ) and returned to control values by 24 hours.

\section{Discussion}

This study does not support the hypothesis that neutrophils have a role in increased airspace epithelial permeability induced by cigarette smoke condensate. Although TNF can induce epithelial permeability, this study also does not provide evidence for a role for TNF in cigarette smoke condensate-induced increased epithelial permeability. However, the data do confirm an association between changes in lung glutathione levels and the increase in epithelial permeability induced by cigarette smoke condensate.

Intratracheal instillation of cigarette smoke condensate produced a consistent recruitment of macrophages and neutrophils into the distal airspaces. This is similar to that which occurs in cigarette smokers, in whom alveolar leucocyte numbers are increased compared with nonsmokers. ${ }^{7}$ The similarities between this model and the events in human smokers in terms of the inflammatory response in the bronchoalveolar lavage and the development of a resolving increase in airspace epithelial permeability justifies the use of this model, which we have described previously ${ }^{11}$ in a study of the mechanisms of the increased epithelial permeability.

The present study shows a coincidence between the peak numbers of neutrophils in BAL fluid and the peak increase in epithelial permeability six hours after instillation of cigarette smoke condensate. Thus neutrophil sequestration, their migration from the pulmonary capillaries through the interstitial space and epithelium, or their presence in the airspaces could contribute to local changes in lung epithelial permeability. Our data clearly show that the abolition of both circulating and airspace neutrophils recruited by instilled cigarette smoke condensate in this model does not attenuate epithelial permeability induced by cigarette smoke condensate in the rat lung.

Hulbert and colleagues ${ }^{22}$ showed that an increase in alveolar permeability occurred before the migration of polymorphonuclear cells to the lung airspaces in smoke-exposed guinea pigs, and that it was restored to normal before the neutrophil infiltration in the lung subsided, suggesting that the increase in permeability induced by smoking does not require the presence of neutrophils.

Induction of a leukopenia attenuated the increase in epithelial permeability induced by inhaling the oxidant gas ozone - a component of cigarette smoke - in one study ${ }^{14}$ but not in others. ${ }^{1213}$ The discrepancy between these data may relate to the complicating effects of the drugs used to induce neutropenia in the airspace epithelium. In our study, however, we used an antineutrophil antibody to induce neutropenia which avoids such confounding effects.

TNF increases the permeability of epithelial cell monolayers in a time-dependent and dosedependent manner. ${ }^{16}$ In the present study, intratracheal instillation of TNF $\alpha$ induced an increase in epithelial permeability in the rat lung. The mechanism is not clear, but may be related to an alteration in epithelial cell tight junctions, as occurs in lungs exposed to nitrogen dioxide. ${ }^{23}$ It is unlikely, however, that TNF plays a part in the increased epithelial permeability induced by cigarette smoke condensate, since the TNF activity in BAL fluid was low compared with the levels needed to induce increased permeability following intratracheal instillation. Furthermore, leucocytes obtained from rats six hours after instillation of cigarette smoke condensate, at the time of maximum epithelial permeability, have a decreased ability to release TNF. This is consistent with the findings of Dubar and coworkers $^{17}$ who showed that cigarette smoke exposure in vitro decreased TNF and IL-6 release from alveolar macrophages obtained from guinea pigs and healthy human subjects. The reduction in TNF release by alveolar macrophages exposed to cigarette smoke may be due to a direct toxic effect on these cells. These results are in contrast to those of Pessina and coworkers ${ }^{24}$ who showed that macrophages 
in BAL fluid from rats exposed to cigarette smoke released more $T N F \alpha$ in culture than control macrophages. However, the gas phase of smoke was used in their study, which may explain the different findings.

Although the small amounts of TNF in the bronchoalveolar space after treatment with cigarette smoke condensate are unlikely to induce epithelial permeability, TNF may participate by causing the neutrophil influx seen in this study, either directly ${ }^{25}$ or by inducing the release of other mediators from resident lung cells which may influence neutrophil chemotaxis. ${ }^{15}$ These include IL- 8 released by epithelial cells which occurs when these cells are stimulated with TNF. ${ }^{26}$ However, TNF antibody had no effect on cigarette smoke condensate-induced epithelial permeability. This was not due to an inactive antibody as we have previously shown the effectiveness of this antibody in blocking both TNF and the increase in epithelial permeability in a rat model of acute alveolitis in vivo. ${ }^{16}$ Thus, there is no evidence to support a role for TNF in the increased epithelial permeability induced by cigarette smoke condensate.

Since cigarette smoke has an enormous oxidant potential, ${ }^{27}$ increased epithelial permeability may result from a direct oxidant effect on epithelial cells. This study shows that intratracheal instillation of cigarette smoke condensate results in a marked fall in lung GSH content one hour after instillation, with a return to control values six hours after instillation associated with a concomitant increase in GSSG. This suggests that some of the GSH has been oxidised. However, the increase in GSSG does not account for all of the fall in GSH so other reactions, such as the formation of GSH adducts with cigarette smoke condensate, are also likely to have occurred. ${ }^{28}$

A dramatic reduction in the $\mathrm{GSH}$ concentration in the BAL fluid occurs 1-6 hours after instillation of cigarette smoke condensate. Furthermore, in previous studies we have shown that a reduction in GSH by inhibition of glutathione synthesis induces increased epithelial permeability in vivo ${ }^{16}$ and in vitro. ${ }^{11}$ The epithelial cells of the alveolar surface of the human lower respiratory tract are vulnerable to oxidants derived from inhaled cigarette smoke. The extent of oxidant-induced injury is dependent on the ability of the host to provide an adequate antioxidant screen. ${ }^{8}$ Cantin and colleagues reported that the epithelial lining fluid of the lower respiratory tract contains large amounts of reduced $\mathrm{GSH}$, with even higher levels in chronic smokers. ${ }^{29}$ However, the acute depletion of GSH in the BAL fluid by cigarette smoke condensate, which we have also shown to occur following acute smoking in $\operatorname{man},{ }^{30}$ may lead to oxidant-induced injury to epithelial cells. Disturbance of cell tight junctions manifest as an increase in epithelial permeability may be the result of such injury.

However, the peak increase in epithelial permeability occurs later than the maximum reduction of total lung GSH. The initial instillation of cigarette smoke condensate presumably causes an acute fall in GSH levels in the lung and BAL fluid followed by a rapid recovery, since the lung tissue has a huge antioxidant potential. However, the total lung GSH content may not be a true reflection of the localised GSH levels in epithelial cells, where there may be a focal depletion at points of maximum deposit/exposure. A study by Voisin and colleagues ${ }^{10}$ showed that the susceptibility of alveolar macrophages to cigarette smoke was related to the intracellular GSH content. The persistent reduction in GSH in the BAL fluid up to six hours after cigarette smoke condensate instillation may represent a sustained epithelial oxidant burden which induces epithelial injury, reflected as an increased epithelial permeability six hours after cigarette smoke condensate instillation. We have previously shown that cigarette smoke condensate induces a fall in intracellular GSH levels in epithelial cells associated with increased epithelial permeability, and that increasing extracellular levels of GSH ameliorates the effect of cigarette smoke condensate on epithelial permeability in vitro. ${ }^{11}$ Furthermore, enhancing the fall in intracellular GSH levels by blocking its synthesis enhances the increase in cigarette smoke condensateinduced epithelial permeability ${ }^{11}$ and injury. ${ }^{31}$

Taken together, the data in this and our previous studies ${ }^{1131}$ present strong evidence to support our hypothesis that reduced glutathione is an important protective mechanism against cigarette smoke-induced airspace epithelial perturbation.

Supported by the Norman Salvesen Emphysema Research Trust and the Tobacco Products Research Trust. The authors wish to thank Eileen Neal for preparing the manuscript.

1 Hogg JC. The effect of smoking on airway permeability. Chest 1983;83:1-2

2 Simani, Inove S, Hogg JC. Penetration of the respiratory epithelium of guinea pigs following exposure to cigarette smoke. Lab Invest 1974;43:81-94.

3 Burns AR, Hosford SP, Dunn LA, Walker DC, Hogg JC. Respiratory epithelial permeability after cigarette smoke exposure in guinea pigs. $\mathcal{F}$ Appl Physiol 1989;66:2109-16.

4 Jones J, Lawler P, Crawley J, Minty B, Hulands G, Veall $\mathrm{N}$. Increased alveolar epithelial permeability in cigarette

smokers. Lancet 1980;1:66-8.
Dusser DJ, Minty BD, Collignon M-A G, Hinge D, Barritault LG, Huchon GJ. Regional respiratory clearance of aerosolized [99m]Tc-DTPA; posture and smoking effect. f Appl Physiol 1986;60:2000-6.

6 Heffner JE, Repine JE. Pulmonary strategies of antioxidant defense. Am Rev Respir Dis 1989;140:531-54.

7 Hoidal JR, Niewoehner DE. Pathogenesis of emphysema. Chest 1983;4:679-85.

8 McCusker K, Hoidal J. Selective increase on antioxidant enzyme activity in the alveolar macrophage from cigarette smokers and smoke-exposed hamsters. Am Rev Respir Dis 1990;141:678-82.

9 Smith LJ, Anderson J. Oxygen-induced lung damage: relationship to lung mitochondrial glutathione levels. $\mathrm{Am}$ Rev Respir Dis 1992;146:1452-7.

10 Voisin C, Aerts C, Fournier E, Firlik M. Acute effects of tobacco smoke on alveolar macrophages cultured in gas phase. Eur $\mathcal{F}$ Respir Dis 1985;66(Suppl 139):76-81.

$11 \mathrm{Li}$ XY, Donaldson K, Rahman I, MacNee W. An investigation of the role of glutathione in the increased epithelial permeability induced by cigarette smoke in vivo and in vitro. Am $\mathcal{F}$ Respir Crit Care Med 1994;149:1518-25.

12 Kleeberger SR, Hudak BB. Acute ozone-induced change in airway permeability: role of infiltrating leukocytes. $\mathcal{f} A p p l$ Physiol 1992;72:670-6.

13 Bassett DJP, Elbon CL, Reichenbaugh SS, Boswell GA, Stevens TM, McGowan MC, et al. Pretreatment with Appl Pharmacol 1989;100:32-40.

14 Bhalla DK, Daniels DS, Luu NT. Attenuation of ozoneinduced airway permeability in rats by pretreatment with cyclophosphamide, FPL 55712, and

15 Strieter RM, Lukacs NW, Standiford TJ, Kunkel SL. Cytokines and lung inflammation: mechanisms of neutrophil
triet kines and lung inflammation: mechanisms of ne
recruitment to the lung. Thorax 1993;48:765-9.

$16 \mathrm{Li} \mathrm{XY,} \mathrm{Donaldson} \mathrm{K,} \mathrm{Brown} \mathrm{D,} \mathrm{MacNee} \mathrm{W.} \mathrm{The} \mathrm{role} \mathrm{of}$ tumour necrosis factor in increased airspace epithelial 
permeability in acute lung inflammation. Am $\mathcal{F}$ Respir Cell Mol Biol 1995;13:185-95.

17 Dubar V, Gosset P, Aerts C, Boisin C, Wallaert B, Tonnel $\mathrm{AB}$. In vitro acute effects of tobacco smoke on tumour necrosis factor alpha and interleukin-6 production by alveolar macrophages. Exp Lung Res 1993;19:345-59.

18 Ringler DH, Dabich L. Hematology and clinical biochemistry. In: Baker HJ, Lindsey JR, Weisbroth SH, eds. The laboratory rat. Volume 1. Biology and disease. London Academic Press, 1979;108-12.

19 Flick DA, Gifford GE. Comparison of in vitro cell cytotoxicity assays for tumour necrosis factor. 7 Immunol Methods 1984;68:167-75.

20 Tietze F. Enzymic method for quantitative determination of nanogram amounts of total and oxidized glutathione: application to mammalian blood and other tissue. Anal Biochem 1969;27:502-22.

21 Griffith OW. Determination of glutathione and glutathione disulfide using glutathione reductase and 2-vinylopyridine. Anal Biochem 1980;106:207-12.

22 Hulbert WC, Walker DC, Jackson A, Hogg JC. Airway permeability to horseradish peroxidase in guinea pigs: th repair phase after injury by cigarette smoke. Am Rev Respi Dis 1981;123:320-6.

23 Gordon RE, Solano D, Kleinerman J. Tight junction alterations of respiratory epithelium following long-term $\mathrm{NO}_{2}$ exposure and recovery. Exp Lung Res 1986;11:17993.
24 Pessina GP, Paulesu L, Coradeschi F, Luzzi E, Stefano AD, Tanzini M, et al. Effects of acute cigarette smoke exposure on macrophage kinetics and release of tumour necrosis factor alpha in rats. Inflammation 1993;2:119-22.

25 Mason MJ, Epps DEV. In vivo neutrophil emigration in response to interleukin-1 and tumour necrosis factor alpha. F Leukocyte Biol 1989;45:62-8.

26 Cromwell O, Hamid O, Corrigan CJ, Barkans J, Meng O Collins PD, et al. Expression and generation of interleukin8, IL-6 and granulocyte-macrophage colony-stimulating factor by bronchial epithelial cells and enhancement by IL- 1 beta and tumour necrosis factor alpha. Immunology 1992;77:330-7.

27 Church $T$, Pryor WA Free radical chemistry of cigarette moke and its toxicological implications. Environ Health Perspect 1985;64:111-26.

28 Rahman I, Li XY, Donaldson K, Harrison DJ, MacNee W. Glutathione homeostasis in alveolar epithelial cells in vitro 13:L285-92.

29 Cantin AM, North SL, Hubbard RC, Crystal RG. Normal alveolar epithelial lining fluid contains high levels of glutathione. $\mathcal{f}$ Appl Physiol 1987;63:152-7.

30 Morrison D, Lannan S, Langridge A, Rahman I, MacNee W. The effect of acute cigarette smoking on epithelial permeability, inflammation and oxidant status in the airpermeability, inflammation and oxidant status in the
spaces of chronic smokers. Eur Respir 7 1994;7:185s.

31 Lannan S, Donaldson K, Brown D, MacNee W. Effect of cigarette smoke and its condensates on alveolar epithelial cell injury in vitro. Am 7 Physiol: Lung Cell Mol Physiol 1994;266:L92-100. 